\title{
Effective mass suppression in a ferromagnetic two-dimensional electron liquid
}

\author{
Reza Asgari, ${ }^{1}$ T. Gokmen, ${ }^{2}$ B. Tanatar, ${ }^{3}$ Medini Padmanabhan, ${ }^{2}$ and M. Shayegan ${ }^{2}$ \\ ${ }^{1}$ School of Physics, Institute for Research in Fundamental Sciences (IPM), 19395-5531 Tehran, Iran \\ ${ }^{2}$ Department of Electrical Engineering, Princeton University, Princeton, New Jersey 08544, USA \\ ${ }^{3}$ Department of Physics, Bilkent University, Bilkent, Ankara 06800, Turkey
}

(Received 19 February 2009; published 22 June 2009)

\begin{abstract}
We present numerical calculations of the electron effective mass in an interacting ferromagnetic twodimensional electron system. We consider quantum interaction effects associated with the charge-density fluctuation-induced many-body vertex corrections. Our theory, which is free of adjustable parameters, reveals that the effective mass is suppressed (relative to its band value) in the strong-coupling limit, in good agreement with recent experimental results.
\end{abstract}

DOI: 10.1103/PhysRevB.79.235324

PACS number(s): 73.20.Mf, 71.10.Ca

\section{INTRODUCTION}

Two-dimensional electron systems (2DESs) realized at semiconductor interfaces are of continuing interest ${ }^{1,2}$ from both basic physics and technological points of view. As a function of the interaction strength, which is characterized by the ratio $r_{s}$ of the Coulomb energy to Fermi energy, many novel correlated ground states have been predicted such as a paramagnetic liquid $\left(r_{s}<26\right)$, ferromagnetic liquid $\left(26<r_{s}<35\right)$, and Wigner crystal $\left(r_{s}>35\right){ }^{3}$ In the paramagnetic-liquid phase, interaction typically leads to an enhancement of effective mass $\left(m^{*}\right)$ and spin susceptibility $\left(\chi^{*} \propto g^{*} m^{*}\right)$, where $g^{*}$ is the Lande $g^{*}$ factor. Effective mass is an important concept in Landau's Fermi-liquid theory since it provides a direct measure of the many-body interactions in the electron system as characterized by increasing $r_{s}$.

The effective mass $m^{*}$ renormalized by interactions has been experimentally studied ${ }^{4-8}$ for various paramagnetic 2DESs as a function of $r_{s}$. In the highly interacting dilute paramagnetic regime $\left(3<r_{s}<26\right), m^{*}$ is typically significantly enhanced compared to its band value $m_{b}$ and tends to increase with increasing $r_{s}{ }^{4-14}$ A question of particular interest is the dependence of $m^{*}$ on the 2D electrons' spin and valley degrees of freedom as these affect the exchange interaction. Recent measurements of $m^{*}$ for 2D electrons confined to AlAs quantum wells (QWs) revealed that, when the 2DES is fully valley and spin polarized, $m^{*}$ is suppressed down to values near or even slightly below $m_{b} \cdot{ }^{8,15,16}$ Note that in these experiments, $r_{s}<22$ so that the $2 \mathrm{DES}$ is in the paramagnetic regime but a strong magnetic field is applied in order to fully spin polarize the electrons. Here we present theoretical calculations indicating that the $m^{*}$ suppression is caused by the absence (freezing out) of the spin fluctuations. The results of our $m^{*}$ calculations are indeed in semiquantitative agreement with the measurements.

Previous theoretical calculations of the effective mass are mostly performed within the framework of Landau's Fermiliquid theory whose key ingredient is the quasiparticle (QP) concept and its interactions. This entails the calculation of effective electron-electron interactions which enter the many-body formalism allowing the calculation of effective mass. A number of works considered different variants of the leading order in the screened interaction for the self-energy ${ }^{10-12,17-22}$ from which density, spin polarization, and temperature dependence of effective mass are obtained. In these calculations the on-shell approximation ${ }^{19-21}$ yields a diverging effective mass but the full solution of the Dyson equation yields only a mild enhancement. ${ }^{10-12}$ Almost all these works considered a paramagnetic 2DES as past experiments concentrated on the effective mass enhancement in partially spin-polarized 2D systems with $r_{s}<26$.

\section{THEORY}

We consider a ferromagnetic 2DES as a model for a system of electronic carriers with band mass $m_{b}$ in a semiconductor heterostructure with dielectric constant $\kappa$. The bare electron-electron interaction is given by $v_{\mathbf{q}}=2 \pi e^{2} /(\kappa q)$. At zero temperature there is only one relevant parameter for the homogeneous, ferromagnetic 2DES, the usual Wigner-Seitz density parameter $r_{s}=\left(\pi n a_{B}^{2}\right)^{-1 / 2}$ in which $a_{B}=\hbar^{2} \kappa /\left(m_{b} e^{2}\right)$ is the Bohr radius in the medium of interest.

The QP self-energy with momentum $\mathbf{k}$ and frequency $\omega$ in a fully polarized electron system can be written as

$$
\begin{aligned}
\Sigma^{\uparrow}(\mathbf{k}, \omega)= & -\int \frac{d^{2} \mathbf{q}}{i(2 \pi)^{2}} v_{\mathbf{q}} \int_{-\infty}^{\infty} \frac{d \Omega}{2 \pi} \frac{1}{\varepsilon(\mathbf{q}, \Omega)} \\
& \times\left[\frac{1-n_{\mathrm{F}}\left(\xi_{\mathbf{k}}^{\uparrow}\right)}{\omega+\Omega-\xi_{\mathbf{k}+\mathbf{q}}^{\uparrow} / \hbar+i \eta}+\frac{n_{\mathrm{F}}\left(\xi_{\mathbf{k}}^{\uparrow}\right)}{\omega+\Omega-\xi_{\mathbf{k}+\mathbf{q}}^{\uparrow} / \hbar-i \eta}\right] .
\end{aligned}
$$

Here $\hat{\xi}_{\mathbf{k}}^{\uparrow}=\varepsilon_{\mathbf{k}}-\varepsilon_{\mathrm{F}}$ where $\varepsilon_{\mathbf{k}}=\hbar^{2} \mathbf{k}^{2} /\left(2 m_{b}\right)$ is the single-particle energy with $\varepsilon_{\mathrm{F}}=\hbar^{2} k_{\mathrm{F}}^{\uparrow 2} /\left(2 m_{b}\right)$ and $k_{\mathrm{F}}^{\uparrow}=\left(4 \pi n_{2 \mathrm{D}}\right)^{1 / 2}=2 /\left(r_{s} a_{B}\right)$, respectively, being the Fermi energy and wave vector; $n_{\mathrm{F}}(k)$ is the Fermi function. In Eq. (1), $\varepsilon(\mathbf{q}, \omega)$ is the dynamical screening function for which we use the form appropriate for a ferromagnetic 2DES derived from Kukkonen-Overhauser effective interaction. ${ }^{23}$ The many-body exchange and correlation effects are introduced through the local-field factors (LFF) $G_{\sigma, \sigma^{\prime}}(q, \omega)\left(\sigma\right.$ and $\sigma^{\prime}$ are spin indices) which take the Pauli-Coulomb hole around a charged particle into account. The dynamical screening function reads 


$$
\frac{1}{\varepsilon(\mathbf{q}, \omega)}=1+v_{\mathbf{q}}\left[1-G_{\uparrow}^{+}(\mathbf{q}, \omega)\right]^{2} \chi_{\mathrm{C}}(\mathbf{q}, \omega),
$$

where $G_{\uparrow}^{+}$is the LFF associated with charge fluctuations. This expression is similar to the Kukkonen and Overhauser interaction $^{23}$ where the spin-fluctuation term is dropped. A similar expression has also been reported in Refs. 24 and 25. In Eq. (2) $\chi_{\mathrm{C}}(\mathbf{q}, \omega)$ represents the density-density response function, which in turn is determined by the local-field factor $G_{\uparrow}^{+}(\mathbf{q}, \omega)$ via the relation

$$
\chi_{\mathrm{C}}(\mathbf{q}, \omega)=\frac{\chi_{\uparrow}^{0}(\mathbf{q}, \omega)}{1-v_{\mathbf{q}}\left[1-G_{\uparrow}^{+}(\mathbf{q}, \omega)\right] \chi_{\uparrow}^{0}(\mathbf{q}, \omega)},
$$

in which $\chi_{\uparrow}^{0}(q, \omega)$ is the density response function of the spin-polarized electrons. The expression for the noninteracting density response function on the imaginary frequency axis is obtained for use in Eq. (3) as

$$
\chi_{\uparrow}^{0}(\mathbf{q}, i \Omega)=\frac{m_{b}^{2}}{2 \pi \hbar^{2} q^{2}}\left(\sqrt{2} \sqrt{a_{\uparrow}+\sqrt{a_{\uparrow}^{2}+\left(\frac{q^{2} \Omega}{\hbar m_{b}}\right)^{2}}}-\frac{q^{2}}{m_{b}}\right),
$$

where we have defined $a_{\uparrow}=q^{4} / 4 m_{b}^{2}-q^{2} k_{\mathrm{F}}^{\uparrow 2} / m_{b}^{2}-\Omega^{2} / \hbar^{2}$. It is evident that setting $G_{\uparrow}^{+}(\mathbf{q}, \omega)=0$, we recover the standard random-phase approximation (RPA). In what follows, we shall make the common approximation of neglecting the frequency dependence of $G_{\uparrow}^{+}$.

Quite generally, once the QP retarded self-energy is known, the QP excitation energy $\delta \mathcal{E}_{\mathrm{QP}}^{\uparrow}(\mathbf{k})$, which is the QP energy measured from the chemical potential $\mu^{\uparrow}$ of the interacting ferromagnetic 2DES, can be calculated by solving self-consistently the Dyson equation

$$
\delta \mathcal{E}_{\mathrm{QP}}^{\uparrow}(\mathbf{k})=\xi_{\mathbf{k}}^{\uparrow}+\left.\Re e \sum_{\text {ret }}^{R}(\mathbf{k}, \omega)\right|_{\omega=\delta \mathcal{E}_{\mathrm{QP}}^{\uparrow}(\mathbf{k}) / \hbar}
$$

Alternatively, the QP excitation energy can also be calculated from

$$
\delta \mathcal{E}_{\mathrm{QP}}^{\uparrow}(\mathbf{k})=\xi_{\mathbf{k}}^{\uparrow}+\left.\Re e \sum_{\text {ret }}^{R}(\mathbf{k}, \omega)\right|_{\omega=\xi_{\mathbf{k}}^{\uparrow} / \hbar}
$$

This is called the on-shell approximation (OSA) and it is $\operatorname{argued}^{26}$ to be a better approach than solving the full Dyson equation since the noninteracting Green's function is used in Eq. (1). Here $\mathfrak{R} e \Sigma_{\text {ret }}^{R}(\mathbf{k}, \omega)$ is defined as $\mathfrak{R} e \Sigma_{\text {ret }}^{\uparrow}(\mathbf{k}, \omega)-\Sigma_{\text {ret }}^{\uparrow}\left(\mathbf{k}_{\mathrm{F}}^{\uparrow}, 0\right)$.

The effective mass $m_{\uparrow}^{*}(k)$ is now calculated from

$$
\frac{1}{m_{\uparrow}^{*}(k)}=\frac{1}{\hbar^{2} k} \frac{d \delta \mathcal{E}_{\mathrm{QP}}^{\uparrow}(k)}{d k},
$$

where for $\delta \mathcal{E}_{\mathrm{QP}}^{\uparrow}$ we have at our disposal the Dyson and OSA approaches. Evaluating $m_{\uparrow}^{*}(k)$ at $k=k_{\mathrm{F}}^{\uparrow}$, one gets the QP effective mass at the Fermi contour. Clearly from Eqs. (2) and (3) LFF is the basic quantity for an evaluation of the QP properties. We have used the parametrized forms of LFFs $G^{+}(q, \zeta)$ and $G^{-}(q, \zeta)$ (and, in particular, $G_{\uparrow}^{+}(q)=G^{+}(q, \zeta=1)$, where $\zeta$ is the spin polarization) of Moreno and Marinescu. ${ }^{27}$

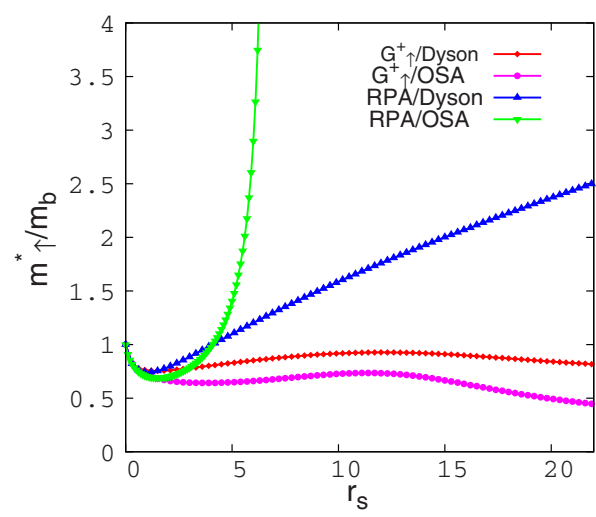

FIG. 1. (Color online) Many-body effective mass as a function of $r_{s}$ for $0 \leq r_{s} \leq 22$ for a ferromagnetic 2DES.

\section{RESULTS AND DISCUSSION}

We now present our numerical results, which are based on the $\operatorname{LFF} G_{\uparrow}^{+}(q)$ as input. In Fig. 1 we show our numerical results of the QP effective mass both in OSA and Dyson approximations. The QP effective mass suppression is substantially smaller in the Dyson equation calculation than in the OSA; the reason is that a significant cancellation occurs between the numerator and the denominator in the effective mass expression in the Dyson approach. To clarify the effect of charge-density fluctuation we have also shown the RPA results which do not take the strong many-body fluctuations into account. Note that the LFF takes into account multiplescattering events to infinite order as compared to the RPA where these effects are neglected. In the limit of small $\zeta$ and $r_{s} \rightarrow 0$, the effective mass can be analytically shown to be $m_{\uparrow}^{*} / m_{b}=1+(1-\zeta / 2.0) r_{s} \ln r_{s} /(\sqrt{2} \pi)$ which our numerical calculations faithfully reproduce.

In Fig. 2 we compare our effective mass calculations with the experimental results. ${ }^{8,15,16}$ The measurements were made on 2DESs confined to modulation-doped AlAs QWs of width 4.5, 11, 12, and $15 \mathrm{~nm}$ (samples A, B, C, and D). These samples were grown on GaAs substrates using molecularbeam epitaxy. In bulk AlAs, electrons occupy three degenerate ellipsoidal conduction-band valleys at the $X$ points of the

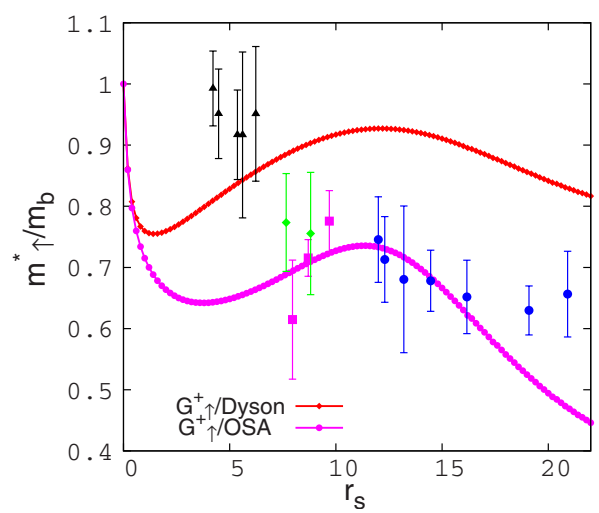

FIG. 2. (Color online) Many-body effective mass as a function of $r_{s}$ for $0 \leq r_{s} \leq 22$ for the ferromagnetic 2DES in comparison to experiments in Refs. 8 and 16. Different symbols denote different samples; triangles: A, squares: $\mathrm{B}$, circles: $\mathrm{C}$, and diamonds: D. 
Brillouin zone with longitudinal and transverse effective masses $m_{l}=1.05$ and $m_{t}=0.205$ (in units of the free electron mass). Thanks to the slightly larger lattice constant of AlAs compared to GaAs, the AlAs QW layer is under biaxial compressive strain. Because of this compression, the 2DES in the wider QW samples (B, C, and D) occupy two in-plane valleys with their major axes lying in the plane. ${ }^{28}$ In our measurements on these samples, we applied uniaxial in-plane strain to break the symmetry between these two valleys so that only one in-plane valley with an anisotropic Fermi contour and band effective mass of $m_{b}=\sqrt{m_{l} m_{t}}=0.46$ is occupied. ${ }^{28}$ In sample A, however, thanks to its very small QW width, the confinement energy of the out-of-plane valley is lower (because of its larger mass along the growth direction) so that the electrons occupy this valley and therefore have an isotropic Fermi contour and band effective mass is $m_{b}=m_{t}=0.205 .^{28}$ The effective masses were deduced from the temperature dependence of the Shubnikov-de Haas oscillations, the details of which are given in Refs. 8, 15, and 16. We emphasize that the data shown here (Fig. 2) were taken on single-valley 2DESs which were subjected to sufficiently large magnetic fields to fully spin polarize the electrons.

It appears in Fig. 2 that the OSA accounts overall for the observed reduction in $m_{\uparrow}^{*}$ below the band value reasonably well. The agreement is particularly good for the wider samples (B, C, and D) which have $r_{s}>7$. The $m^{*}$ data for the narrowest sample (A), however, fall above the theoretical predictions. We do not know the reason for this discrepancy. However, we point out that, besides the difference in the shapes of the Fermi contour, there is another difference between sample A and the other three samples. Because of the very narrow width of sample A's quantum well and the prevalence of interface roughness scattering, ${ }^{29}$ the mobility of the electrons in this sample is much lower (about a factor of 6) than in other samples for comparable $r_{s}$. It is possible that the higher disorder in sample A is responsible for $m^{*}$ being larger; this conjecture is indeed consistent with the results of calculations ${ }^{10}$ which predict a larger $m^{*}$ for more disordered samples.

From Figs. 1 and 2 we draw two main conclusions. (i) The RPA and present results are rather similar in the weakcoupling limit $\left(r_{s}<1\right)$. (ii) In the strong-coupling regime $\left(r_{s}>3\right)$, however, our theoretical calculations which incorporate the proper many-body effects exhibit a mass suppression, similar to the experimental data, while the RPA results show a mass enhancement and are far from the experimental data. We emphasize that the effective mass at the Fermi contour is significantly suppressed in the fully polarized case because of the absence of spin-fluctuation contribution. This suppression suggests that the antisymmetric Landau parameter $F_{1}^{a}<0$ and thus higher angular momentum Landau parameters may be negligible in a fully spin-polarized 2DES.

To gain further insight to the density dependence of $m^{*}$, we have calculated the on-shell effective mass as a function of particle momentum $k$ using Eq. (7) evaluated at $\omega(k)=\xi_{\mathrm{k}}^{\uparrow} / \hbar$ and $r_{s}=5$. More specifically, we use

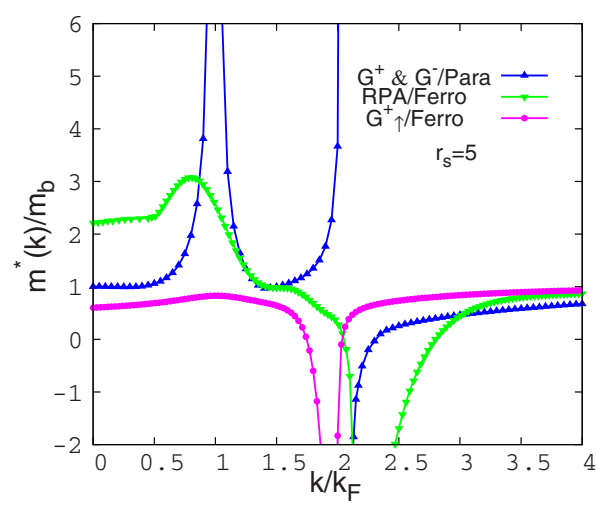

FIG. 3. (Color online) Many-body on-shell effective mass as a function of $k / k_{\mathrm{F}}$ at $r_{s}=5$ for 2DES with the combined effect of charge fluctuations in comparison to paramagnetic 2DES.

$$
\frac{m_{b}}{m_{\uparrow}^{*}(k)}=1+\frac{m_{b}}{\hbar^{2} k} \frac{d}{d k} \Re e \Sigma_{\mathrm{ret}}^{\uparrow}\left(k, \xi_{k}^{\uparrow}\right)
$$

for a ferromagnetic case. The results for both paramagnetic and ferromagnetic cases are shown in Fig. 3. $m^{*}(k)$ for a paramagnetic 2 DES by using $G^{+}(q, \zeta=0)$ and $G^{-}(q, \zeta=0)$ has a sharp peak around $k \approx k_{\mathrm{F}}$ where $k_{\mathrm{F}}=2 /\left(r_{s} a_{B}\right)$ and a resonancelike divergent behavior around $k \approx 2 k_{\mathrm{F}}$. The peak around $k_{\mathrm{F}}$ is associated with spin fluctuations and the divergent behavior around $2 k_{\mathrm{F}}$ is related to density fluctuations. ${ }^{22,24}$ In particular, the latter divergence has been extensively studied by Zhang et al. ${ }^{22}$ within the RPA. It is related to the dispersion instability and coincides with the plasmon emission. $m_{\uparrow}^{*}(k)$ for the ferromagnetic 2DES, on the other hand, clearly shows the disappearance of the peak associated with spin fluctuations. Thus, $m_{\uparrow}^{*}(k)$ is very weakly momentum dependent for $k<k_{\mathrm{F}}^{\uparrow}$, since there is a substantial cancellation between the residue and the exchange plus line self-energy contribution in this regime which make the real part of the retarded self-energy approximately linear with respect to $k .{ }^{11}$ The divergence associated with charge fluctuations is still present, showing a negative peak around $k=2 k_{\mathrm{F}} . m_{\uparrow}^{*}(k)$ calculated within the RPA reproduces quantitatively the divergent behavior associated with charge fluctuations but shows some structure for $k \leq k_{\mathrm{F}}^{\uparrow}$, therefore failing to account for the absence of spin fluctuations. Our densitydependent effective mass results (Figs. 1 and 2) are consistent with $m^{*}(k)$ calculations which we have checked for a range of $r_{s}$ values.

We have also calculated the renormalization factor $Z_{\uparrow}\left(r_{s}\right)$ which is equal to the discontinuity in the momentum distribution at $k_{\mathrm{F}}$ and defined by $Z_{\uparrow}^{-1}=1-\left.\hbar^{-1} \partial_{\omega} \Re e \Sigma_{\text {ret }}^{\uparrow}(k, \omega)\right|_{k=k_{\text {, }}^{\uparrow} \omega=0}$. The effect of charge fluctuations is to make the $Z_{\uparrow}$ values larger at large $r_{s}$ compared to the case when they are not included as shown in Fig. 4. This means that charge-density fluctuations tend to stabilize the system whereas the RPA works in the opposite direction. ${ }^{11}$ In the present case including the LFF helps preserve the Fermi-liquid picture in the low-density regime.

We have performed our numerical calculations for strictly 2DES. As indicated above, experimental samples have a fi- 


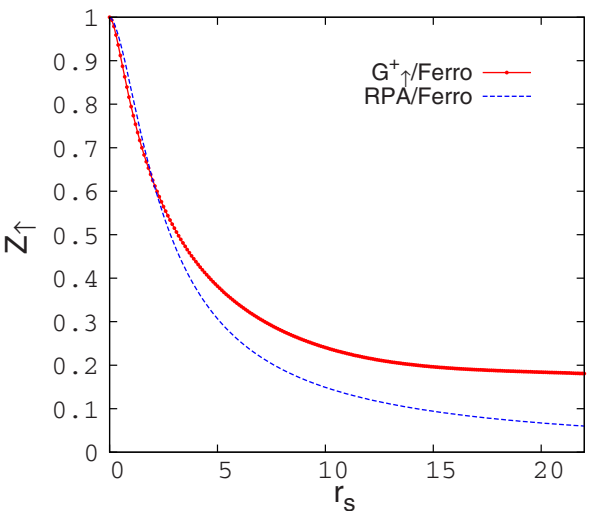

FIG. 4. (Color online) Renormalization constant $Z_{\uparrow}$ as a function of $r_{s}$ for $0<r_{s}<22$ for a ferromagnetic 2DES.

nite thickness in the range of 5-15 nm. Our theoretical model may be extended to include the finite quantum well width effects in the following manner. Choosing, say, an infinite square-well model with width $L$ will modify the bare Coulomb interaction, $v_{\mathbf{q}} \rightarrow v_{\mathbf{q}} F(q L)$, in which $F(x)$ is a form factor. ${ }^{30}$ For consistency, one should also calculate the localfield factor $G_{\uparrow}^{+}(q)$ using the same model for the finite width effects. This would provide a better comparison with experiments. In our case, the local-field factor we use was constructed $^{27}$ by the quantum Monte Carlo data for a strictly 2DES and it is not straightforward to incorporate the finite with effects within such an approach. Previous calculations ${ }^{12}$ of the effective mass for a paramagnetic 2DES suggest that the effect of a finite thickness is to suppress $m^{*}$. Therefore, we surmise that a similar qualitative effect would occur for the ferromagnetic 2DES. On the other hand, the finite temperature and disorder effects have a tendency to enhance the effective mass ${ }^{10,21}$ which may lead to a cancellation. These issues require a more systematic study.

\section{SUMMARY}

In conclusion, our theoretical calculations incorporating the proper Pauli-Coulomb hole and multiscattering processes show that in an interacting fully spin-polarized 2DES the absence of spin fluctuations reduces the effective mass below its band value, in agreement with experimental data. Our results also demonstrate the inadequacy of RPA to account for the observed effective mass suppression.

\section{ACKNOWLEDGMENTS}

R.A. thanks M. Polini for helpful discussions. The work at Princeton University was supported by the NSF. B.T. was supported by TUBITAK (Grant No. 108T743) and TUBA.
${ }^{1}$ T. Ando, A. B. Fowler, and F. Stern, Rev. Mod. Phys. 54, 437 (1982).

${ }^{2}$ G. F. Giuliani and G. Vignale, Quantum Theory of the Electron Liquid (Cambridge University Press, Cambridge, England, 2005).

${ }^{3}$ C. Attaccalite, S. Moroni, P. Gori-Giorgi, and G. B. Bachelet, Phys. Rev. Lett. 88, 256601 (2002); B. Tanatar and D. M. Ceperley, Phys. Rev. B 39, 5005 (1989).

${ }^{4}$ J. L. Smith and P. J. Stiles, Phys. Rev. Lett. 29, 102 (1972).

${ }^{5}$ W. Pan, D. C. Tsui, and B. L. Draper, Phys. Rev. B 59, 10208 (1999).

${ }^{6}$ A. A. Shashkin, S. V. Kravchenko, V. T. Dolgopolov, and T. M. Klapwijk, Phys. Rev. B 66, 073303 (2002).

${ }^{7}$ Y.-W. Tan, J. Zhu, H. L. Stormer, L. N. Pfeiffer, K. W. Baldwin, and K. W. West, Phys. Rev. Lett. 94, 016405 (2005).

${ }^{8}$ M. Padmanabhan, T. Gokmen, N. C. Bishop, and M. Shayegan, Phys. Rev. Lett. 101, 026402 (2008).

${ }^{9}$ Y. Kwon, D. M. Ceperley, and R. M. Martin, Phys. Rev. B 50, 1684 (1994); M. Holzmann, B. Bernu, V. Olevano, R. M. Martin, and D. M. Ceperley, ibid. 79, 041308(R) (2009).

${ }^{10}$ R. Asgari, B. Davoudi, and B. Tanatar, Solid State Commun. 130, 13 (2004).

${ }^{11}$ R. Asgari, B. Davoudi, M. Polini, G. F. Giuliani, M. P. Tosi, and G. Vignale, Phys. Rev. B 71, 045323 (2005).

${ }^{12}$ R. Asgari and B. Tanatar, Phys. Rev. B 74, 075301 (2006).

${ }^{13} \mathrm{~S}$. Gangadharaiah and D. L. Maslov, Phys. Rev. Lett. 95, 186801 (2005).

${ }^{14}$ Y. Zhang and S. Das Sarma, Phys. Rev. Lett. 95, 256603 (2005).

${ }^{15}$ T. Gokmen, M. Padmanabhan, and M. Shayegan, Phys. Rev.
Lett. 101, 146405 (2008).

${ }^{16}$ T. Gokmen, M. Padmanabhan, K. Vakili, E. Tutuc, and M. Shayegan, Phys. Rev. B 79, 195311 (2009).

${ }^{17}$ I. K. Marmorkos and S. Das Sarma, Phys. Rev. B 44, 3451 (1991); H.-J. Schulze, P. Schuck, and N. Van Giai, ibid. 61, 8026 (2000).

${ }^{18}$ S. Yarlagadda and G. F. Giuliani, Phys. Rev. B 49, 7887 (1994); 61, 12556 (2000); C. S. Ting, T. K. Lee, and J. J. Quinn, Phys. Rev. Lett. 34, 870 (1975).

${ }^{19}$ H. M. Böhm and K. Schörkhuber, J. Phys.: Condens. Matter 12, 2007 (2000).

${ }^{20}$ Y. Zhang and S. Das Sarma, Phys. Rev. B 71, 045322 (2005).

${ }^{21}$ S. Das Sarma, Victor M. Galitski, and Ying Zhang, Phys. Rev. B 69, 125334 (2004).

${ }^{22}$ Y. Zhang, V. M. Yakovenko, and S. Das Sarma, Phys. Rev. B 71, 115105 (2005).

${ }^{23}$ C. A. Kukkonen and A. W. Overhauser, Phys. Rev. B 20, 550 (1979).

${ }^{24}$ T.-K. Ng and K. S. Singwi, Phys. Rev. B 34, 7743 (1986).

${ }^{25}$ S. Tanaka and S. Ichimaru, Phys. Rev. B 39, 1036 (1989).

${ }^{26}$ T. M. Rice, Ann. Phys. (N.Y.) 31, 100 (1965).

${ }^{27}$ J. Moreno and D. C. Marinescu, Phys. Rev. B 68, 195210 (2003).

${ }^{28}$ M. Shayegan, E. P. De Poortere, O. Gunawan, Y. P. Shkolnikov, E. Tutuc, and K. Vakili, Phys. Status Solidi B 243, 3629 (2006).

${ }^{29}$ K. Vakili, Y. P. Shkolnikov, E. Tutuc, E. P. De Poortere, M. Padmanabhan, and M. Shayegan, Appl. Phys. Lett. 89, 172118 (2006).

${ }^{30}$ A. Gold, Phys. Rev. B 35, 723 (1987). 